\title{
Bounds on third Hankel determinant for certain classes of analytic functions
}

\author{
Jugal K. Prajapat, Deepak Bansal and Sudhananda Maharana
} Abstract. In this paper, the estimate for the third Hankel determinant $H_{3,1}(f)$
of Taylor coefficients of function $f(z)=z+\sum_{n=2}^{\infty} a_{n} z^{n}$, belonging to certain classes
of analytic functions in the open unit disk $\mathbb{D}$, are investigated.

Mathematics Subject Classification (2010): 30C45, 30C50.

Keywords: Analytic, starlike and convex functions, Fekete-Szegö functional, Hankel determinants.

\section{Introduction}

Let $\mathcal{H}(\mathbb{D})$ denote the class of analytic functions in the open unit disk

$$
\mathbb{D}=\{z \in \mathbb{C}:|z|<1\}
$$

and $\mathcal{A}$ be the class of functions $f \in \mathcal{H}(\mathbb{D})$, having the form

$$
f(z)=z+\sum_{n=2}^{\infty} a_{n} z^{n}, \quad z \in \mathbb{D},
$$

with the standard normalization $f(0)=0, f^{\prime}(0)=1$. We denote by $\mathcal{S}$, the subclass of $\mathcal{A}$ consisting of functions which are also univalent in $\mathbb{D}$, and $\mathcal{P}$ denotes the class of functions $p \in \mathcal{H}(\mathbb{D})$ with $\Re(p(z))>0, z \in \mathbb{D}$.

A function $f \in \mathcal{A}$ is called starlike (with respect to origin 0 ), if $f$ is univalent in $\mathbb{D}$ and $f(\mathbb{D})$ is a starlike domain. We denote this class of starlike functions by $\mathcal{S}^{*}$. A function $f \in \mathcal{S}$ maps the unit disk $\mathbb{D}$ onto a convex domain is called convex function, and this class of functions is denoted by $\mathcal{K}$. Let $\mathcal{M}(\lambda)$ be the subclass of $\mathcal{A}$ consisting of functions $f(z)$ which satisfy the inequality

$$
\Re\left(\frac{z f^{\prime}(z)}{f(z)}\right)<\lambda, \quad z \in \mathbb{D},
$$


for some $\lambda(\lambda>1)$. And let $\mathcal{N}(\lambda)$ be the subclass of $\mathcal{A}$ consisting of functions $f(z)$ if and only if $z f^{\prime}(z) \in \mathcal{M}(\lambda)$, i.e. $f(z)$ satisfy the inequality

$$
\Re\left(1+\frac{z f^{\prime \prime}(z)}{f^{\prime}(z)}\right)<\lambda, \quad z \in \mathbb{D},
$$

for some $\lambda(\lambda>1)$. These classes $\mathcal{M}(\lambda)$ and $\mathcal{N}(\lambda)$ were investigated recently by Nishiwaki and Owa [19] (see also [23]). For $1<\lambda \leq 4 / 3$, the classes $\mathcal{M}(\lambda)$ and $\mathcal{N}(\lambda)$ were investigated by Uralegaddi et al. [32].

Throughout the present paper, by $\mathcal{M}$ we always mean the class of functions $\mathcal{M}(3 / 2)$, and by $\mathcal{N}$ we always mean the class of functions $\mathcal{N}(3 / 2)$. Ozaki [24] proved that functions in $\mathcal{N}$ are univalent in $\mathbb{D}$. Moreover, if $f \in \mathcal{N}$, then (see e.g. [11, Theorem 1] and [21, p. 196]) one have

$$
\frac{z f^{\prime}(z)}{f(z)} \prec g(z)=\frac{2(1-z)}{2-z}, \quad z \in \mathbb{D},
$$

where $\prec$ denotes the subordination [18]. We see that $g$ above is univalent in $\mathbb{D}$ and maps $\mathbb{D}$ onto the disk $|w-(2 / 3)|<2 / 3$. Thus, functions in $\mathcal{M}$ are starlike in $\mathbb{D}$.

For $f \in \mathcal{A}$ of the form (1.1), a classical problem settled by Fekete and Szegö [9] is to find the maximum value of the coefficient functional $\Phi_{\lambda}(f):=a_{3}-\lambda a_{2}^{2}$ for each $\lambda \in[0,1]$, over the function $f \in \mathcal{S}$. By applying the Löewner method they proved that

$$
\max _{f \in \mathcal{S}}\left|\Phi_{\lambda}(f)\right|= \begin{cases}1+2 \exp \left(\frac{-2 \lambda}{1-\lambda}\right), & \lambda \in[0,1), \\ 1, & \lambda=1 .\end{cases}
$$

The problem of calculating the maximum of the coefficient functional $\Phi_{\lambda}(f)$ for various compact subfamilies of $\mathcal{A}$, as well as $\lambda$ being an arbitrary real or complex number, has been studied by many authors (see e.g. $[1,12,13,17,30,31]$ ).

We denote by $H_{q, n}(f)$ where $n, q \in \mathbb{N}=\{1,2, \cdots\}$, the Hankel determinant of functions $f \in \mathcal{A}$ of the form (1.1), which is defined by

$$
H_{q, n}(f)=\left|\begin{array}{cccc}
a_{n} & a_{n+1} & \cdots & a_{n+q-1} \\
a_{n+1} & a_{n+2} & \cdots & a_{n+q} \\
\vdots & \vdots & \vdots & \vdots \\
a_{n+q-1} & a_{n+q} & \cdots & a_{n+2(q-1)}
\end{array}\right| \quad\left(a_{1}=1\right)
$$

The Hankel determinant $H_{q, n}(f)$ has been studied by several authors including Cantor [6], Noonan and Thomas [20], Pommerenke [26, 25], Hayman [10], Ehrenborg [8], which are useful, in showing that a function of bounded characteristic in $\mathbb{D}$.

Indeed, $H_{2,1}(f)=\Phi_{1}(f)$ is the Fekete-Szegö coefficient functional. Many authors have studied the problem of calculating $\max _{f \in \mathcal{F}}\left|H_{2,2}(f)\right|$ for various subfamily $\mathcal{F}$ of the class $f \in \mathcal{A}$ (see e.g. [2, 4, 14]). Recently, several authors including Babalola [3], Bansal et al. [5], Prajapat et al. [28], Raza and Malik [29] have obtained the bounds on the third Hankel determinant $H_{3,1}(f)$ for certain families of analytic functions, 
which is defined by

$$
\begin{aligned}
H_{3,1}(f) & =\left|\begin{array}{lll}
a_{1} & a_{2} & a_{3} \\
a_{2} & a_{3} & a_{4} \\
a_{3} & a_{4} & a_{5}
\end{array}\right| \\
& =a_{3}\left(a_{2} a_{4}-a_{3}^{2}\right)-a_{4}\left(a_{4}-a_{2} a_{3}\right)+a_{5}\left(a_{3}-a_{2}^{2}\right) .
\end{aligned}
$$

In the present paper, we investigate the bounds on $H_{3,1}(f)$ for the functions belonging to the classes $\mathcal{M}$ and $\mathcal{N}$ defined above. In order to get the main results, we need the following known results.

Lemma 1.1. ([16]) If $p \in \mathcal{P}$ be of the form $p(z)=1+\sum_{n=1}^{\infty} c_{n} z^{n}$, then

$$
2 c_{2}=c_{1}^{2}+x\left(4-c_{1}^{2}\right)
$$

and

$$
4 c_{3}=c_{1}^{3}+2 c_{1} x\left(4-c_{1}^{2}\right)-c_{1} x^{2}\left(4-c_{1}^{2}\right)+2\left(4-c_{1}^{2}\right)\left(1-|x|^{2}\right) z,
$$

for some $x, z$ with $|x| \leq 1$ and $|z| \leq 1$.

Lemma 1.2. ([22, Theorem 1]) If $f \in \mathcal{N}$ be given by (1.1), then

$$
\left|a_{n}\right| \leq \frac{1}{n(n-1)}, \quad n \geq 2 .
$$

The result is sharp for the function $f_{n}$ such that $f_{n}^{\prime}(z)=\left(1-z^{n-1}\right)^{1 /(n-1)}, n \geq 2$.

As it is known that, if $f(z) \in \mathcal{N}$ then $z f^{\prime}(z) \in \mathcal{M}$, therefore from Lemma 1.2, we conclude that

Lemma 1.3. If $f(z) \in \mathcal{M}$ be given by (1.1), then

$$
\left|a_{n}\right| \leq \frac{1}{n-1}, \quad n \geq 2 .
$$

The result is sharp for the function $g_{n}(z)=z\left(1-z^{n-1}\right)^{1 /(n-1)}, n \geq 2$.

Lemma 1.4. ([22, Corollary 2]) If $f \in \mathcal{N}$ be given by (1.1), then

$$
\left|a_{3}-a_{2}^{2}\right| \leq 1 / 4 \text {. }
$$

Equality is attained for the function $f$ such that $f^{\prime}(z)=\left(1-z^{2} e^{i \theta}\right)^{1 / 2}, \theta \in[0,2 \pi]$.

\section{Main results}

Our first main result is contained in the following theorem:

Theorem 2.1. Let the function $f \in \mathcal{M}$ be given by (1.1), then

$$
\left|a_{3}-a_{2}^{2}\right| \leq 1
$$

The result (2.1) is sharp and equality in (2.1) is attained for the function

$$
e_{1}(z)=z-z^{2}
$$


Proof. If the function $f \in \mathcal{M}$ be given by (1.1), then we may write

$$
\frac{z f^{\prime}(z)}{f(z)}=\frac{3}{2}-\frac{1}{2} p(z)
$$

where $p(z)=1+\sum_{n=1}^{\infty} c_{n} z^{n}$ is analytic in $\mathbb{D}$ and $\Re(p(z))>0$ in $\mathbb{D}$. Also, we have $\left|c_{n}\right| \leq 2$ for all $n \geq 1$ (see [7]). In terms of power series expansion, the last identity is equivalent to

$$
\sum_{n=1}^{\infty} n a_{n} z^{n}=\left(1-\frac{1}{2} \sum_{n=1}^{\infty} c_{n} z^{n}\right)\left(\sum_{n=1}^{\infty} a_{n} z^{n}\right)
$$

where $a_{1}=1$. Equating the coefficients of $z^{n}$ on both sides, we deduce that

$$
a_{2}=-\frac{1}{2} c_{1}, \quad a_{3}=\frac{1}{8}\left(c_{1}^{2}-2 c_{2}\right), \quad a_{4}=\frac{1}{48}\left(6 c_{1} c_{2}-8 c_{3}-c_{1}^{3}\right) .
$$

Now using Lemma 1.1 for some $x$ such that $|x| \leq 1$, we have

$$
\left|a_{3}-a_{2}^{2}\right|=\left|\frac{1}{8}\left(c_{1}^{2}-2 c_{2}\right)-\frac{1}{4} c_{1}^{2}\right|=\frac{1}{8}\left|2 c_{1}^{2}+x\left(4-c_{1}^{2}\right)\right| .
$$

As $\left|c_{1}\right| \leq 2$, taking $c_{1}=c$, assume without restriction that $c \in[0,2]$. Hence applying the triangle inequality with $\mu=|x|$, we obtain

$$
\begin{aligned}
\left|a_{3}-a_{2}^{2}\right| & \leq \frac{1}{8}\left[2 c^{2}+\mu\left(4-c^{2}\right)\right] \\
& =F_{1}(c, \mu) .
\end{aligned}
$$

Let $\Omega=\{(c, \mu): 0 \leq c \leq 2$ and $0 \leq \mu \leq 1\}$. Differentiating $F_{1}$ with respect to $\mu$, we get

$$
\frac{\partial F_{1}}{\partial \mu}=\frac{1}{8}\left(4-c^{2}\right) \geq 0 \text { for } \quad 0 \leq \mu \leq 1 .
$$

Therefore $F_{1}(c, \mu)$ is a non-decreasing function of $\mu$ on the closed interval $[0,1]$. Thus, it attains maximum value at $\mu=1$. Let

$$
\max _{0 \leq \mu \leq 1} F_{1}(c, \mu)=F_{1}(c, 1)=\frac{c^{2}+4}{8}=G_{1}(c) .
$$

We observe that $G_{1}(c)$ is an increasing function in $[0,2]$, so it will attains maximum value at $c=2$. Next, to find the critical point on the boundary of $\Omega$, we examine all the four line segments of $\Omega$. Along the line segment $c=2$ with $0 \leq \mu \leq 1$, we have $F_{1}(c, \mu)=F_{1}(2, \mu)=1$, which is a constant, thus every point on the line segment is the critical point. For the line segment $c=0$ with $0 \leq \mu \leq 1$, we have $F_{1}(c, \mu)=F_{1}(0, \mu)=\mu / 2$. For the line segment $\mu=0$ with $0 \leq c \leq 2$, we have $F_{1}(c, \mu)=F_{1}(c, 0)=c^{2} / 4$, which gives the critical point $(0,0)$ and $F_{1}(0,0)=0$. Also, for the line segment $\mu=1$ with $0 \leq c \leq 2$, we have $F_{1}(c, \mu)=F_{1}(c, 1)=\left(c^{2}+4\right) / 8$, which gives another critical point $(0,1)$ and $F_{1}(0,1)=1 / 2$.

Putting this all together we can conclude that the maximum of $F_{1}(c, \mu)$ lie at each point along the line segment $c=2$ with $0 \leq \mu \leq 1$, which can also be verified 
through the mathematica plot of $F_{1}(c, \mu)$ over the region $\Omega$ given below in the Figure 1 . Hence

$$
\max _{\Omega} F_{1}(c, \mu)=F_{1}(2, \mu)=1
$$

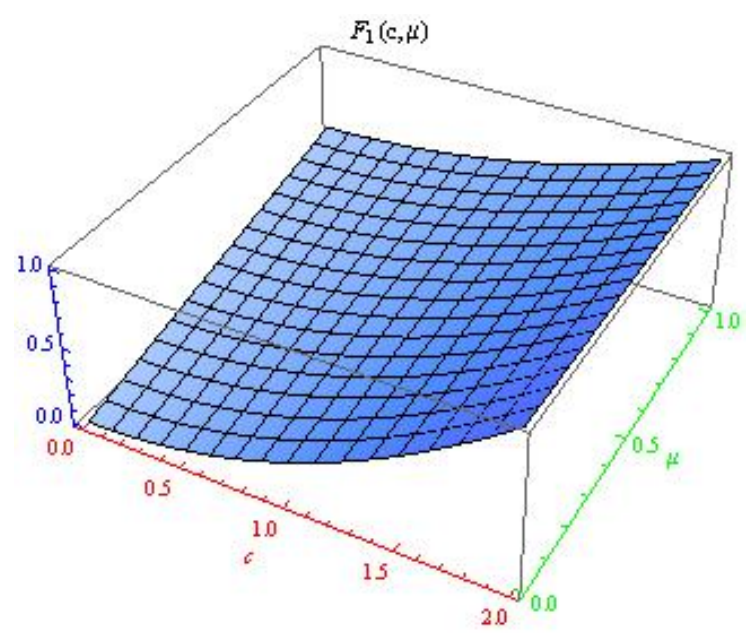

FiguRE 1. Mapping of $F_{1}(c, \mu)$ over $\Omega$

To find the extremal function, setting $c_{1}=2$ and $x=1$ in Lemma 1.1, we find that $c_{2}=c_{3}=2$, using these values in (2.3), we get that $a_{2}=-1$ and $a_{3}=a_{4}=0$, therefore the extremal function would be $e_{1}(z)=z-z^{2}$. A simple calculation shows that $e_{1}(z) \in \mathcal{M}$. This complete the proof of Theorem 2.1.

Theorem 2.2. Let the function $f \in \mathcal{M}$ be given by (1.1), then

$$
\left|a_{2} a_{4}-a_{3}^{2}\right| \leq \frac{1}{4}
$$

The result (2.4) is sharp and equality is attained for the function

$$
e_{2}(z)=z-\frac{1}{2} z^{3} \text { and } e_{3}(z)=z\left(1-z^{2}\right)^{1 / 2}
$$

Proof. Using (2.3) and applying Lemma 1.1 for some $x$ and $z$ such that $|x| \leq 1$ and $|z| \leq 1$, we have

$$
\begin{aligned}
\left|a_{2} a_{4}-a_{3}^{2}\right|= & \left|-\frac{1}{96} c_{1}\left(6 c_{1} c_{2}-8 c_{3}-c_{1}^{3}\right)-\frac{1}{64}\left(c_{1}^{2}-2 c_{2}\right)^{2}\right| \\
= & \frac{1}{192} \mid-3 x^{2}\left(4-c_{1}^{2}\right)^{2}+2 c_{1}^{2} x\left(4-c_{1}^{2}\right)-4 c_{1}^{2} x^{2}\left(4-c_{1}^{2}\right) \\
& +8 c_{1}\left(4-c_{1}^{2}\right)\left(1-|x|^{2}\right) z \mid .
\end{aligned}
$$


As $\left|c_{1}\right| \leq 2$, taking $c_{1}=c$, assume without restriction that $c \in[0,2]$. Thus applying the triangle inequality with $\mu=|x|$, we obtain

$$
\begin{aligned}
\left|a_{2} a_{4}-a_{3}^{2}\right| & \leq \frac{1}{192}\left[\left(4-c^{2}\right)\left\{3 \mu^{2}\left(4-c^{2}\right)+2 c^{2} \mu+4 \mu^{2} c^{2}+8 c\left(1-\mu^{2}\right)\right\}\right] \\
& =\frac{1}{192}\left[\left(4-c^{2}\right)\left\{\left(12-8 c+c^{2}\right) \mu^{2}+2 c^{2} \mu+8 c\right\}\right] \\
& =F_{2}(c, \mu) .
\end{aligned}
$$

Differentiating $F_{2}(c, \mu)$ in the above equation with respect to $\mu$, we get

$$
\frac{\partial F_{2}}{\partial \mu}=\frac{\left(4-c^{2}\right)}{96}\left\{\left(12-8 c+c^{2}\right) \mu+c^{2}\right\} \geq 0 \quad \text { for } \quad 0 \leq \mu \leq 1 .
$$

Therefore $F_{2}(c, \mu)$ is a non-decreasing function of $\mu$ on closed interval $[0,1]$. Thus, it attains maximum value at $\mu=1$. Let

$$
\max _{0 \leq \mu \leq 1} F_{2}(c, \mu)=F_{2}(c, 1)=\frac{16-c^{4}}{64}=G_{2}(c) .
$$

We observe that $G_{2}(c)$ is a decreasing function in $[0,2]$, so it will attains maximum value at $c=0$. Next, to find the critical point on the boundary of $\Omega$, we examine all the four line segments of $\Omega$ by the earlier method used in Theorem 2.1, and we are getting $(0,0),(2 / \sqrt{3}, 0)$ and $(0,1)$ are the critical points and $F_{2}(0,0)=0, F_{2}(2 / \sqrt{3}, 0)=$ $2 / 9 \sqrt{3}$ and $F_{2}(0,1)=1 / 4$. Therefore maximum value of $F_{2}(c, \mu)$ is obtained by putting $c=0$ and $\mu=1$, which can also verified through the mathematica plot of $F_{2}(c, \mu)$ over $\Omega$ given below in Figure 2. Hence

$$
\max _{\Omega} F_{2}(c, \mu)=F_{2}(0,1)=\frac{1}{4} .
$$

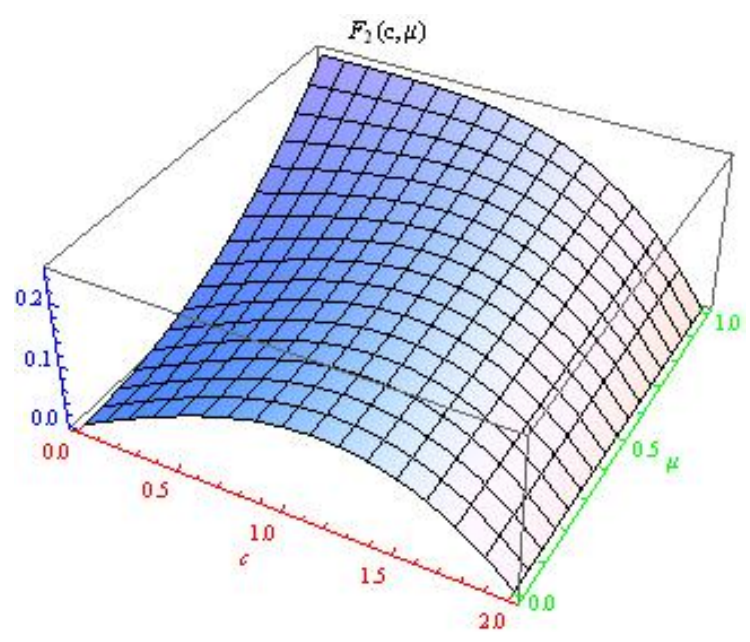

Figure 2. Mapping of $F_{2}(c, \mu)$ over $\Omega$ 
Now, to find extremal function, set $c_{1}=0$ and selecting $x=1$ in Lemma 1.1, we find that $c_{2}=2$ and $c_{3}=0$. Using these values in (2.3), we get $a_{2}=a_{4}=0$ and $a_{3}=$ $1 / 2$, therefore one of the extremal function of (2.4) would be $e_{2}(z)=z-\frac{1}{2} z^{3}$. We can also see that equality in $(2.4)$ is attended for the function $e_{3}(z)=z\left(1-z^{2}\right)^{1 / 2} \in \mathcal{M}$. A simple calculation shows that $e_{2} \in \mathcal{M}$ and $e_{3} \in \mathcal{M}$. This complete the proof of Theorem 2.2.

Theorem 2.3. Let the function $f \in \mathcal{M}$ be given by (1.1), then

$$
\left|a_{2} a_{3}-a_{4}\right| \leq \frac{2 \sqrt{3}}{9} .
$$

Proof. Using (2.3) and applying Lemma 1.1 for some $x$ and $z$ such that $|x| \leq 1$ and $|z| \leq 1$, we have

$$
\begin{aligned}
\left|a_{2} a_{3}-a_{4}\right| & =\left|\frac{1}{16} c_{1}\left(c_{1}^{2}-2 c_{2}\right)+\frac{1}{48}\left(6 c_{1} c_{2}-8 c_{3}-c_{1}^{3}\right)\right| \\
& =\frac{1}{24}\left|2 c_{1} x\left(4-c_{1}^{2}\right)-c_{1} x^{2}\left(4-c_{1}^{2}\right)+2\left(4-c_{1}^{2}\right)\left(1-|x|^{2}\right) z\right| .
\end{aligned}
$$

As $\left|c_{1}\right| \leq 2$, letting $c_{1}=c$, assume without restriction that $c \in[0,2]$. Hence applying the triangle inequality with $\mu=|x|$, we obtain

$$
\begin{aligned}
\left|a_{2} a_{3}-a_{4}\right| & \leq \frac{\left(4-c^{2}\right)}{24}\left[2+2 c \mu+(c-2) \mu^{2}\right] \\
& =F_{3}(c, \mu) .
\end{aligned}
$$

To find the maximum of $F_{3}$ over the region $\Omega$, differentiating $F_{3}$ with respect to $\mu$ and $c$, we get

$$
\begin{aligned}
& \frac{\partial F_{3}}{\partial \mu}=\frac{\left(4-c^{2}\right)}{12}[c+(c-2) \mu] \\
& \frac{\partial F_{3}}{\partial c}=\frac{1}{24}\left[-4 c+\left(8-6 c^{2}\right) \mu+\left(4+4 c-3 c^{2}\right) \mu^{2}\right] .
\end{aligned}
$$

A critical point of $F_{3}(c, \mu)$ must satisfy $\frac{\partial F_{3}}{\partial \mu}=0$ and $\frac{\partial F_{3}}{\partial c}=0$. The condition $\frac{\partial F_{3}}{\partial \mu}=0$ gives $c= \pm 2$ or $\mu=-c /(c-2)$. The interior point $(c, \mu)$ of $\Omega$ satisfying such condition in only $(0,0)$, and at that point $(0,0)$, we have

$$
\left(\frac{\partial^{2} F_{3}}{\partial \mu^{2}}\right)\left(\frac{\partial^{2} F_{3}}{\partial c^{2}}\right)-\left(\frac{\partial^{2} F_{3}}{\partial c \partial \mu}\right)^{2}=0
$$

Hence, it is not certain that at $(0,0)$ function have maximum value in $\Omega$. Since $\Omega$ is closed and bounded and $F_{3}$ is continuous, the maximum of $F_{3}$ shall be attained on the boundary of $\Omega$. Along the line segment $c=2$ with $0 \leq \mu \leq 1$, we have $F_{3}(c, \mu)=F_{3}(2, \mu)=0$, which is a constant. For the line segment $c=0$ with $0 \leq$ $\mu \leq 1$, we have $F_{3}(c, \mu)=F_{3}(0, \mu)=\left(1-\mu^{2}\right) / 3$, which gives the same critical point $(0,0)$ and $F_{3}(0,0)=1 / 3$. For the line segment $\mu=0$ with $0 \leq c \leq 2$, we have $F_{3}(c, \mu)=F_{3}(c, 0)=\left(4-c^{2}\right) / 12$, which gives the same critical point $(0,0)$. Also, for the line segment $\mu=1$ with $0 \leq c \leq 2$, we have $F_{3}(c, \mu)=F_{3}(c, 1)=\left(4 c-c^{3}\right) / 8$, 
which gives another critical point $(2 / \sqrt{3}, 1)$ on this line and $F_{3}(2 / \sqrt{3}, 1)=2 \sqrt{3} / 9$. Therefore, the point $(0,0)$ and $(2 / \sqrt{3}, 1)$ are the only critical points of $F_{3}$ over $\Omega$. Hence, the largest value of $F_{3}(c, \mu)$ over the region $\Omega$ lies at $(2 / \sqrt{3}, 1)$ and

$$
\max _{\Omega} F_{3}(c, \mu)=F_{3}(2 / \sqrt{3}, 1)=\frac{2 \sqrt{3}}{9} .
$$

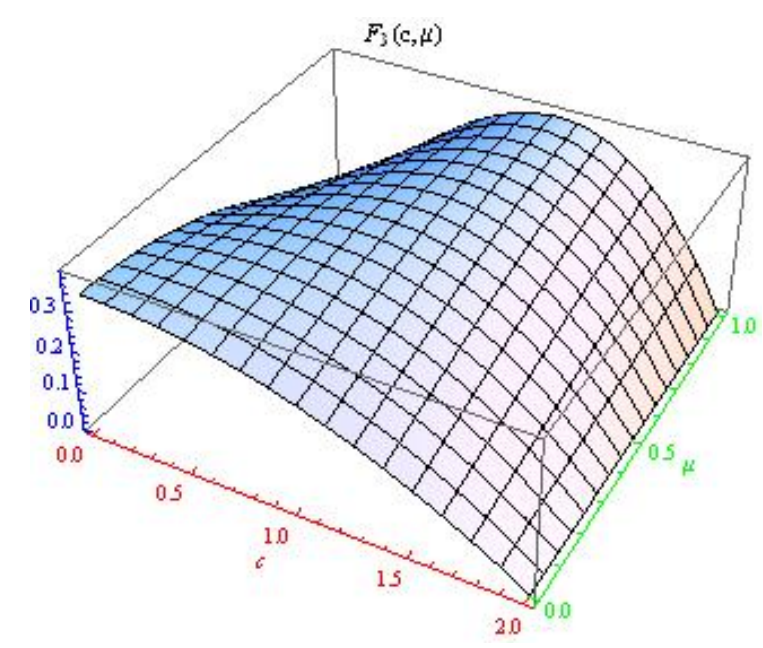

FiguRE 3. Mapping of $F_{3}(c, \mu)$ over $\Omega$

Theorem 2.4. Let the function $f \in \mathcal{M}$ be given by (1.1), then

$$
\left|H_{3,1}(f)\right| \leq \frac{81+16 \sqrt{3}}{216} .
$$

Proof. Using Lemma 1.3, Theorem 2.1, Theorem 2.2, Theorem 2.3 and the triangle inequality on $H_{3,1}(f)$, we get

$$
\begin{aligned}
\left|H_{3,1}(f)\right| & \leq\left|a_{3}\right|\left|a_{2} a_{4}-a_{3}^{2}\right|+\left|a_{4}\right|\left|a_{2} a_{3}-a_{4}\right|+\left|a_{5}\right|\left|a_{3}-a_{2}^{2}\right| \\
& \leq \frac{1}{2} \cdot \frac{1}{4}+\frac{1}{3} \cdot \frac{2 \sqrt{3}}{9}+\frac{1}{4} \cdot 1=\frac{81+16 \sqrt{3}}{216} .
\end{aligned}
$$

This completes the proof of Theorem 2.4.

Theorem 2.5. Let the function $f \in \mathcal{N}$ be given by (1.1), then

$$
\left|a_{2} a_{3}-a_{4}\right| \leq \frac{1}{12}
$$

The result (2.8) is sharp and equality in (2.8) is attained for the function $e_{4}$ where $e_{4}^{\prime}(z)=\left(1-z^{3}\right)^{1 / 3}$. 
Proof. Let the function $f \in \mathcal{N}$ be given by (1.1), then by definitions it is clear that $f(z) \in \mathcal{N}$ if and only if $z f^{\prime}(z) \in \mathcal{M}$, thus replacing $a_{n}$ by $n a_{n}$ in (2.3), we get

$$
a_{2}=-\frac{1}{4} c_{1}, \quad a_{3}=\frac{1}{24}\left(c_{1}^{2}-2 c_{2}\right), \quad a_{4}=\frac{1}{192}\left(6 c_{1} c_{2}-8 c_{3}-c_{1}^{3}\right) .
$$

Now using (2.9) and applying Lemma 1.1 for some $x$ and $z$ such that $|x| \leq 1$ and $|z| \leq 1$, we have

$$
\begin{aligned}
\left|a_{2} a_{3}-a_{4}\right| & =\left|-\frac{1}{96} c_{1}\left(c_{1}^{2}-2 c_{2}\right)-\frac{1}{192}\left(6 c_{1} c_{2}-8 c_{3}-c_{1}^{3}\right)\right| \\
& =\frac{1}{192}\left|3 c_{1} x\left(4-c_{1}^{2}\right)-2 c_{1} x^{2}\left(4-c_{1}^{2}\right)+4\left(4-c_{1}^{2}\right)\left(1-|x|^{2}\right) z\right| .
\end{aligned}
$$

As $\left|c_{1}\right| \leq 2$, taking $c_{1}=c$, assume without restriction that $c \in[0,2]$. Hence applying the triangle inequality with $\mu=|x|$, we obtain

$$
\begin{aligned}
\left|a_{2} a_{3}-a_{4}\right| & \leq \frac{\left(4-c^{2}\right)}{192}\left[4+3 c \mu+2(c-2) \mu^{2}\right] \\
& =F_{4}(c, \mu) .
\end{aligned}
$$

Following the earlier method used in Theorem 2.3, we can show that the global maximum of $F_{4}(c, \mu)$ over the region $\Omega$ is achieved at $(0,0)$ and $F_{4}(0,0)=1 / 12$. This can also be verified through the mathematica plot of $F_{4}(c, \mu)$ over $\Omega$ given below in Figure 4.

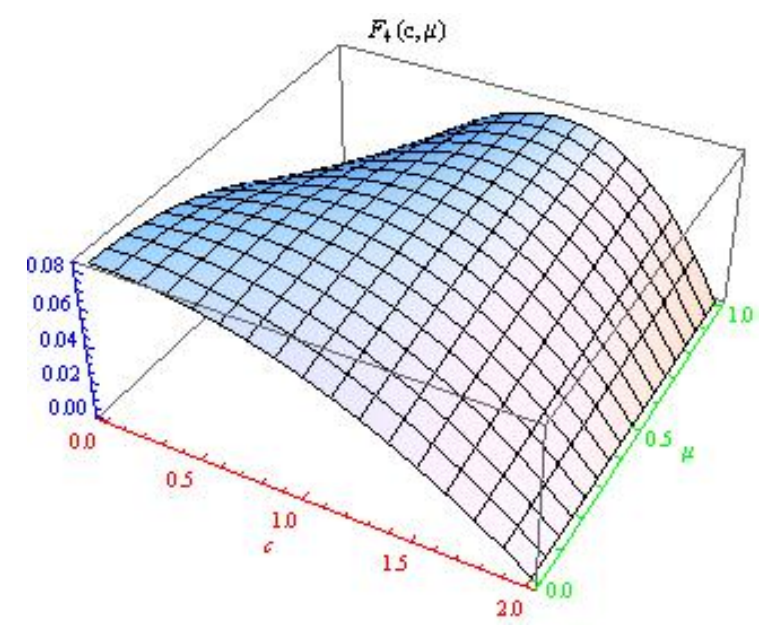

FiguRE 4. Mapping of $F_{4}(c, \mu)$ over $\Omega$

Also observe that equality in (2.8) is attained for the function $e_{4}$ where

$$
e_{4}^{\prime}(z)=\left(1-z^{3}\right)^{1 / 3} \text {. }
$$

A computation shows that $e_{4} \in \mathcal{N}$. Hence the result is obtained. 
Theorem 2.6. Let the function $f \in \mathcal{N}$ be given by (1.1), then

$$
\left|a_{2} a_{4}-a_{3}^{2}\right| \leq \frac{9}{320}
$$

Proof. Using (2.9) and applying Lemma 1.1 for some $x$ and $z$ such that $|x| \leq 1$ and $|z| \leq 1$, we have

$$
\begin{aligned}
\left|a_{2} a_{4}-a_{3}^{2}\right| & =\frac{1}{192}\left|-\frac{1}{4} c_{1}\left(6 c_{1} c_{2}-8 c_{3}-c_{1}^{3}\right)-\frac{1}{3}\left(c_{1}^{2}-2 c_{2}\right)^{2}\right| \\
& =\frac{1}{192}\left|\frac{1}{12} c_{1}^{4}+\frac{1}{6} c_{1}^{2} c_{2}+\frac{4}{3} c_{2}^{2}-2 c_{1} c_{3}\right| \\
& =\frac{1}{2304} \mid 3 x c_{1}^{2}\left(4-c_{1}^{2}\right)-6 x^{2} c_{1}^{2}\left(4-c_{1}^{2}\right)+12 z c_{1}\left(4-c_{1}^{2}\right)\left(1-|x|^{2}\right) \\
& -4\left(4-c_{1}^{2}\right)^{2} x^{2} \mid .
\end{aligned}
$$

As $\left|c_{1}\right| \leq 2$, taking $c_{1}=c$, assume without restriction that $c \in[0,2]$. Thus applying the triangle inequality with $\mu=|x|$, we obtain

$$
\begin{aligned}
\left|a_{2} a_{4}-a_{3}^{2}\right| & \leq \frac{\left(4-c^{2}\right)}{2304}\left\{12 c+3 c^{2} \mu+2\left(8-6 c+c^{2}\right) \mu^{2}\right\} \\
& =F_{5}(c, \mu) .
\end{aligned}
$$

Differentiating $F_{5}(c, \mu)$ with respect to $\mu$, we get

$$
\frac{\partial F_{5}}{\partial \mu}=\frac{\left(4-c^{2}\right)}{2304}\left\{4 \mu\left(c^{2}-6 c+8\right)+3 c^{2}\right\} \geq 0 \quad \text { for } \quad 0 \leq \mu \leq 1 .
$$

Therefore $F_{5}(c, \mu)$ is a non-decreasing function of $\mu$ on closed interval $[0,1]$. Thus, it attains maximum value at $\mu=1$. Let

$$
\max _{0 \leq \mu \leq 1} F_{5}(c, \mu)=F_{5}(c, 1)=\frac{1}{2304}\left(64+4 c^{2}-5 c^{4}\right)=G_{5}(c) .
$$

We can see that $G_{5}(c)$ is an increasing function in $[0, \sqrt{2 / 5}]$, so $G_{5}(c)$ attains maximum value at $c=\sqrt{2 / 5}$. Next, to find the critical points on the boundary of $\Omega$, we examine all the four line segments of $\Omega$ by the earlier method used in Theorem 2.1 and 2.3 , and we get $(0,0),(2 / \sqrt{3}, 0)$ and $(0,1)$ are the critical points and $F_{5}(0,0)=0$, $F_{5}(2 / \sqrt{3}, 0)=1 / 36 \sqrt{3}$ and $F_{5}(0,1)=1 / 36$. Therefore $F_{5}(c, \mu)$ have maximum value at $\mu=1$ and $c=\sqrt{2 / 5}$ in the region $\Omega$. Thus

$$
\max _{\Omega} F_{5}(c, \mu)=F_{5}(\sqrt{2 / 5}, 1)=\frac{9}{320} .
$$

This completes the proof of Theorem 2.6.

Remark 2.7. For $f \in \mathcal{S}$, Thomas [27, p. 166] conjectured that

$$
\left|H_{2, n}(f)\right|=\left|a_{n} a_{n+2}-a_{n+1}^{2}\right| \leq 1, \quad n=2,3,4 \cdots .
$$

Subsequently, Li and Srivastava [15, p. 1040] shown that this conjecture is not valid for $n \geq 4$, i.e. conjecture is valid only for $n=2,3$. From Theorem 2.6 , we found that, if function $f$ is member of class $\mathcal{N}$ and having form (1.1), then $\left|H_{2,2}(f)\right| \leq 9 / 320$. 


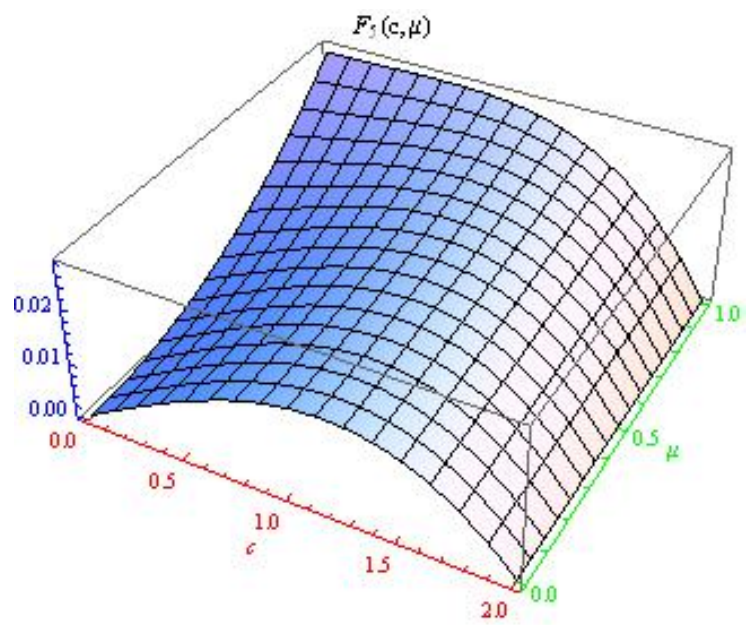

FiguRE 5. Mapping of $F_{5}(c, \mu)$ over $\Omega$

Since all functions in $\mathcal{N}$ are univalent in $\mathbb{D}$. Therefore, Theorem 2.6 validates the Thomas conjecture when $n=2$ for the function belonging to the classes $\mathcal{N}$.

Theorem 2.8. Let the function $f \in \mathcal{N}$ be given by (1.1), then

$$
\left|H_{3,1}(f)\right| \leq \frac{139}{5760} .
$$

Proof. Using Lemma 1.2, Lemma 1.4, Theorem 2.5, Theorem 2.6 and the triangle inequality on $H_{3,1}(f)$, we get

$$
\begin{aligned}
\left|H_{3,1}(f)\right| & \leq\left|a_{3}\right|\left|a_{2} a_{4}-a_{3}^{2}\right|+\left|a_{4}\right|\left|a_{2} a_{3}-a_{4}\right|+\left|a_{5}\right|\left|a_{3}-a_{2}^{2}\right| \\
& \leq \frac{1}{6} \frac{9}{320}+\frac{1}{12} \frac{1}{12}+\frac{1}{20} \frac{1}{4}=\frac{139}{5760} .
\end{aligned}
$$

This completes the proof of Theorem 2.8 .

\section{References}

[1] Abdet Gawad, H.R., Thomas, D.K., The Fekete-Szegö problem for strongly close-toconvex functions, Proc. Amer. Math. Soc., 114(1992), 345-349.

[2] Al-Abbadi, M.H., Darus, M., Hankel determinant for certain class of analytic function defined by generalized derivative operators, Tamkang J. Math., 43(2012), 445-453.

[3] Babalola, K.O., On third order Hankel determinant for some classes of univalent functions, Inequal. Theory Appl., 6(2010), 1-7.

[4] Bansal, D., Upper bound of second Hankel determinant for a new class of analytic functions, Appl. Math. Lett., 26(2013), 103-107.

[5] Bansal, D., Maharana, S., Prajapat, J.K., Third order Hankel determinant for certain univalent functions, J. Korean Math. Soc., 52(2015), no. 6, 1139-1148. 
[6] Cantor, D.G., Power series with the integral coefficients, Bull. Amer. Math. Soc., 69(1963), 362-366.

[7] Duren, P.L., Univalent Functions, Springer Verlag, New Yark Inc. 1983.

[8] Ehrenborg, R., The Hankel determinant of exponential polynomials, Amer. Math. Monthly, 107(2000), 557-560.

[9] Fekete, M., Szegö, G., Eine Benberkung uber ungerada Schlichte funktionen, J. Lond. Math. Soc., 8(1933), 85-89.

[10] Hayman, W.K., On second Hankel determinant of mean univalent functions, Proc. Lond. Math. Soc., 18(1968), 77-94.

[11] Jovanovic, I., Obradovic̀, M., A note on certain classes of univalent functions, Filomat, $\mathbf{9}(1995), 69-72$.

[12] Keogh, F.R., Merkes, E.P., A Coefficient Inequality for Certain Classes of Analytic Functions, Proc. Amer. Math. Soc., 20(1969), 8-12.

[13] Koepf, W., On the Fekete-Szegö problem for close-to-convex functions, Proc. Amer. Math. Soc., 101(1987), 85-95.

[14] Lee, S.K., Ravichandran, V., Supramaniam, S., Bounds for the second Hankel determinant of certain univalent functions, J. Inequal. Appl., 2013(2013), art. 281.

[15] Li, J.L., Srivastava, H.M., Some questions and conjectures in the theory of univalent functions, Rocky Mountain J. Math., 28(1998), 1035-1041.

[16] Libera, R.J., Zlotkiewicz, E.J., Early coefficients of the inverse of a regular convex function, Proc. Amer. Math. Soc., 85(1982), 225-230.

[17] London, R.R., Fekete-Szegö inequalities for close-to-convex functions, Proc. Amer. Math. Soc., 117(1993), 947-950.

[18] Miller, S.S., Mocanu, P.T., Differential Subordinations. Theory and Applications, New York and Basel, Marcel Dekker, 2000.

[19] Nishiwaki, J., Owa, S., Coefficient inequalities for certain analytic functions, Int. J. Math. Math. Sci., 29(2002), 285-290.

[20] Noonan, J.W., Thomas, D.K., On the second Hankel determinant of areally mean pvalent functions, Trans. Amer. Math. Soc., 223(1976), 337-346.

[21] Obradović, M., Ponnusamy, S., Injectivity and starlikeness of section of a class of univalent functions, Contemporary Math., 591(2013), 195-203.

[22] Obradović, M., Ponnusamy, S., Wirths, K.-J., Coefficient characterizations and sections for some univalent functions, Sib. Math. J., 54(2013), 679-696.

[23] Owa, S., Srivastava, H.M., Some generalized convolution properties associated with certain subclasses of analytic functions, J. Inequal. Pure Appl. Math., 3(2002), no. 3, art. 42, 13 pages.

[24] Ozaki, S., On the theory of multivalent functions II, Sci. Rep. Tokyo Bunrika Daigaku. Sect A, 4(1941), 45-87.

[25] Pommerenke, C., On the coefficients and Hankel determinant of univalent functions, J. Lond. Math. Soc., 41(1966), 111-122.

[26] Pommerenke, C., On the Hankel determinant of univalent functions, Mathematika, 14(1967), 108-112.

[27] Parvatham, R., Ponnusamy, S., (eds.) New trends in geometric function theory and application, World Scientific Publishing Company, Singapore, New Jersey, London and Hong Kong, 1981. 
[28] Prajapat, J.K., Bansal, D., Singh, A., Mishra, A.K., Bounds on third Hankel determinant for close-to-convex functions, Acta Univ. Sapientiae Math., 7(2015), no. 2, 210-219.

[29] Raza, M., Malik, S.N., Upper bound of the third Hankel determinant for a class of analytic functions related with Lemniscate of Bernoulli, J. Inequal. Appl., 2013(2013), art. 412.

[30] Shanmugam, T.N., Ramachandran, C., Ravichandran, V., Fekete-Szegö problem for subclass of starlike functions with respect to symmetric points, Bull. Korean Math. Soc., 43(2006), no. 3, 589-598.

[31] Srivastava, H.M., Mishra, A.K., Das, M.K., Fekete-Szegö problem for a subclass of closeto-convex functions, Complex Var. Theory Appl., 44(2001), 145-163.

[32] Uralegaddi, B.A., Ganigi, M.D., Sarangi, S.M., Univalent functions with positive coeffcients, Tamkang J. Math., 25(1994), 225-230.

Jugal K. Prajapat

Department of Mathematics

Central University of Rajasthan

NH-8, Bandarsindri, Kishangarh-305817

Dist.-Ajmer, Rajasthan, India

e-mail: jkprajapat@gmail.com

Deepak Bansal

Department of Mathematics

Govt. College of Engineering and Technology

Bikaner-334004, Rajasthan, India

e-mail: deepakbansal_79@yahoo.com

Sudhananda Maharana

Department of Mathematics

Central University of Rajasthan

NH-8, Bandarsindri, Kishangarh-305817

Dist.-Ajmer, Rajasthan, India

e-mail: snmmath@gmail.com 